\title{
Thermal Behaviour and Glass Transition Dynamics of Inclusion Complexes of $\alpha$-Cyclodextrin with Poly(D,L-lactic acid)
}

\author{
João F. Mano*
}

Inclusion complexes (ICs) have been prepared by the host-guest interaction between $\alpha$-cyclodextrin $(\alpha \mathrm{CD})$ and poly(D,L-lactic acid) (PDLLA). This enables transformation of the amorphous polymer into a well organized channel-type crystalline structure, as studied by wide angle X-ray scattering (WAXS). WAXS experiments using synchrotron radiation allowed the evolution of this crystalline structure to be followed upon heating. The diffraction peaks disappeared above $320^{\circ} \mathrm{C}$, in a temperature region similar to the occurrence of thermal degradation, also investigated by thermogravimetric analysis. No glass transition could be detected in the IC using differential scanning calorimetry (DSC), but non-conventional dynamic mechanical analysis measurements revealed the existence of loss factor peaks shifted to higher temperatures when compared with PDLLA. The relaxation plot of the IC was characterized by an Arrhenius behaviour with a high activation energy that could be consistent with the high geometrical confinement felt by the chains in the nanostructured organization.

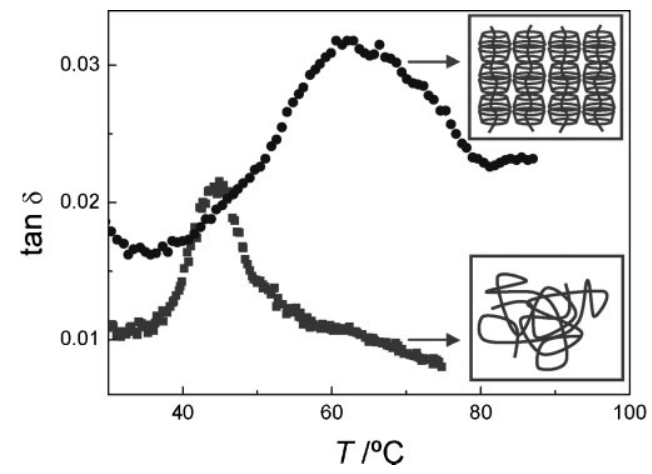

\section{Introduction}

Supramolecular chemistry has opened new possibilities to design highly complex materials through intermolecular non-covalent interactions between their components. ${ }^{[1]}$ In this framework, a variety of inclusion complexes (ICs) has been investigating, especially using cyclodextrins (CDs) as host molecules. ${ }^{[2]} \mathrm{CDs}$ are cyclic molecules that contain six $(\alpha-)$, seven $(\beta-)$, or eight $\left(\gamma^{-}\right) \mathrm{D}(+)$-glucose units joined by $\alpha-1,4$-glycosidic linkages. They exhibit a shallow truncated

J. F. Mano

3B's Research Group - Biomaterials, Biodegradables and Biomimetics, Dept. of Polymer Engineering, University of Minho, Campus de Gualtar, 4710-057 Braga, Portugal IBB - Institute for Biotechnology and Bioengineering, Braga, Portugal

E-mail: jmano@dep.uminho.pt cone shape and a hydrophobic cavity that is apolar relative to the outer surface, being adequate to act as a host for a variety of small and long guest molecules. For the particular case of macromolecules, the penetration of the chains into the empty cavity of the CDs and the corresponded rearrangement of the nanostructure can lead to well organized inclusion complexes (ICs). ${ }^{[3]}$ ICs have been produced with biodegradable aliphatic polyesters, such as poly(e-caprolactone), ${ }^{[4-6]}$ poly(lactic acid), ${ }^{[6-8]}$ or poly(butylene succinate) ${ }^{[5]}$ Such polymers have been proposed for a variety of applications, especially in the environmental and biomedical fields. In particular poly(lactic acid)s have been especially investigated and different enantiomeric forms can be synthesized as a result of the chiral nature of acid lactic. Ohya et al., for example, reported that $\alpha$-CD recognizes such chirality: ${ }^{[7]}$ ICs of $\alpha-C D$ may be easily formed with poly(L-lactic acid), PLLA, whereas the IC formation efficiency with poly(D-lactic 
acid), PDLA, is very low. However, to our knowledge, there is no reports on the formation of ICs of CDs with the racemic copolymer of D-lactic acid and L-lactic acid, poly(D,L-lactic acid), PDLLA, a very well known amorphous polyester used in several biomedical applications, including in the processing of scaffolds for tissue engineering, or in systems for the controlled release of drugs. ${ }^{[9,10]}$ It would be interesting, both from a fundamental and a practical point of view, to produce highly structured materials based on PDLLA, a pure amorphous polymer. It was suggested that polycarbonate, alone or together with poly(methyl methacrylate) could form ICs with $\gamma$-CD. ${ }^{[11-13]}$ However, although organized ICs can be formed with pure polycarbonate, the polymer alone can also crystallize partially. ${ }^{[13]}$ In this work we demonstrate that a pure amorphous polymer, PDLLA, may be transformed into a 'crystalline' structure and its thermal stability will be analyzed in real time using wide angle X-ray diffraction (WAXS). Moreover, a question arises about the conformational mobility of such an organized structure. How could the confinement imposed by the nanostructured IC organization influence or prevent the glass transition dynamics of PDLLA? This issue may be included in the general problem dealing with the molecular dynamics in materials that are geometrically confined in a nanometric spatial scale, ${ }^{[14]}$ such as three-dimensional confinement (such as nanoporous glasses or zeolites), two-dimensional confinement (in silicate layers of nanocomposites or in the layered block copolymers), or thin polymer films and coatings. In PLLA, the effect of confinement was studied by $\mathrm{DSC}^{[15,16]}$ and dielectric relaxation spectroscopy, ${ }^{[17]}$ on samples with different levels of crystallinity. As found in other semi-crystalline polymers, the amorphous component confined between the crystalline lamellae exhibits a constrained dynamics as compared with the conformational mobility in the bulk. In this context, it would be relevant to investigate the presumably greater confinement effect imposed by the IC organization on the glass transition dynamics. For this purpose non-conventional dynamic mechanical analysis measurements will be performed using powdered material.

\section{Experimental Part}

The medical grade PDLLA used in this work was obtained from Purac. The molecular weights of the polymer were $\bar{M}_{\mathrm{n}}=31750$ and $\bar{M}_{\mathrm{w}}=100000 . \alpha$-CD and the organic solvents used were purchased from Aldrich and were used without further purification.

For the preparation of the IC a solution at $50^{\circ} \mathrm{C}$ of PDLLA ( $0.5 \mathrm{~g}$ in $200 \mathrm{~mL}$ of dioxane) was slowly added (drop wise) into a solution of $\alpha$-CD (3.4 g in $25 \mathrm{~mL}$ of distilled water) also at $50{ }^{\circ} \mathrm{C}$ and in continuous agitation. After this step the mixture was agitated for another hour at $50{ }^{\circ} \mathrm{C}$. The heating was then stopped and the stirring was maintained for another $24 \mathrm{~h}$. Finally, the obtained suspension was filtered and the white powder was washed several times with dioxane and distilled water.

The temperature dependence of the PDLLA $/ \alpha C D$ IC crystalline structure was analysed by WAXS using synchrotron radiation $(\lambda=0.15 \mathrm{~nm})$ at the Soft Condensed Matter A2 beamline of HASYLAB (DESY) synchrotron facility in Hamburg (Germany). The sample was heated fast in a special oven, until $80^{\circ} \mathrm{C}$, after which measurements were collected, with exposure times of $20 \mathrm{~s}$, each for $2 \mathrm{~min}$, at different temperatures up to $350^{\circ} \mathrm{C}$. The WAXS angle was calibrated using a poly(ethylene teraphthalate) (PET) standard. More precise X-ray measurements at room temperature were carried out with a Philips PW 1710 diffractometer (The Netherlands) with a $\mathrm{Cu} \mathrm{K} \alpha$ radiation $(\lambda=0.154 \mathrm{~nm})$.

Thermal degradation experiments were also carried out by thermogravimetric analysis (TGA) using a Perkin-Elmer TGA7 apparatus, where the weight of the samples were monitored during heating scans at $5^{\circ} \mathrm{C} \cdot \mathrm{min}^{-1}$. The dynamic mechanical analysis (DMA) experiments were performed using a TRITEC2000B DMA from Triton Technology (UK). Isochronal experiments were performed on metallic pockets that enveloped the IC or PDLLA films, always during heating scans using the single cantilever mode. The material was placed over one half of the flat pocket. The other half was then folded over the material and the ensemble was subjected to a pressure to ensure a close encapsulation of the material within the metallic pocket. During DMA both storage modulus, $E^{\prime}$, and loss factor, $\tan \delta$, were recorded as a function of temperature. Just the second parameter will be of interest and will provide information about relaxation processes occurring in the material; $E^{\prime}$ will essentially measure the elastic modulus of the metallic pocket. Differential scanning calorimetry (DSC) experiments were performed in a 0100 from TA instruments using indium for the calibrations. In order to detect more clearly the glass transition the samples were aged for $14 \mathrm{~d}$ at $40^{\circ} \mathrm{C}^{[16]}$ Scans were then performed from 0 to $80^{\circ} \mathrm{C}$ at $10^{\circ} \mathrm{C} \cdot \mathrm{min}^{-1}$. The samples were then cooled immediately at $20^{\circ} \mathrm{C} \cdot \mathrm{min}^{-1}$ down to $0{ }^{\circ} \mathrm{C}$ and a second heating scan was performed, also at $10^{\circ} \mathrm{C} \cdot \mathrm{min}^{-1}$.

\section{Results and Discussion}

The obtained inclusion complex of $\alpha \mathrm{CD}$ and PDLLA, PDLLA/ $\alpha \mathrm{CD}$, exhibited a very well organised structure at the sub-nanometre level (see inset of Figure 1a). For PDLLA no crystalline peaks were observed (data not shown). During the preparation of the IC, channel-type structures were developed, which is consistent with the strong diffraction peak found at around $2 \theta=20^{\circ[18]}$ where the polymeric chains adopt an extended conformation and are included in a well organized lattice of CD molecules. More precisely, the prominent peaks detected at $7.5^{\circ}(100), 13.0^{\circ}$ (110), and 19.9 (210) are very similar to those observed in inclusion complexes of $\alpha$-CD with poly( $\varepsilon$-caprolactone)s with different molecular architectures, and are consistent with an hexagonal unit cell. ${ }^{[19]}$ Thus we consider that the $\alpha$-CDs are closely packed along a PDLLA chain in the vertical direction (single IC strands) and the single IC strands are hexagonally aligned in the horizontal direction. The thermal 


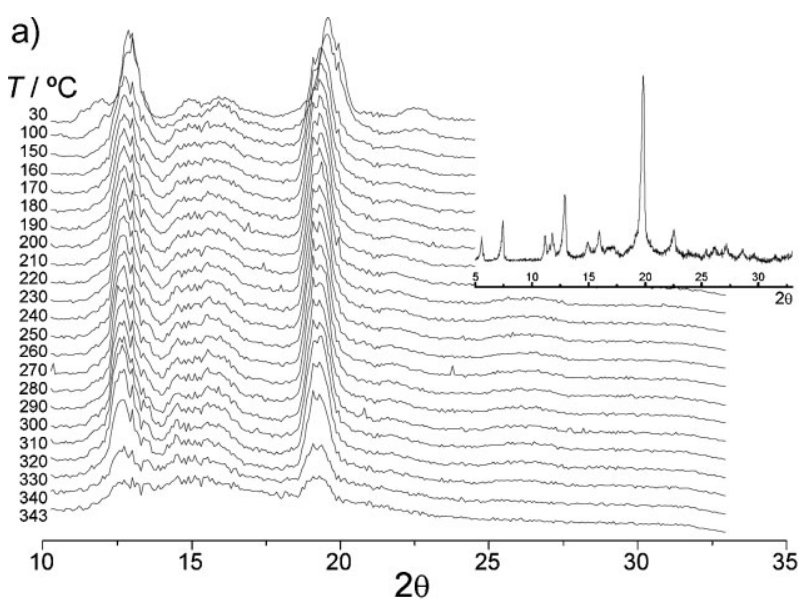

b)

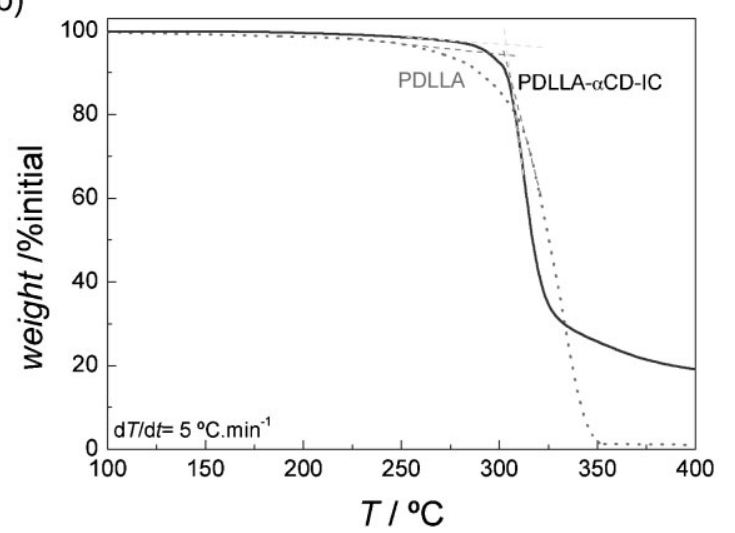

Figure 1. a) Time-resolved WAXS profiles of PDLLA $/ \alpha C D$ during heating using synchrotron radiation. The inset shows the initial diffractogram of the material taken at room temperature using the Philips equipment. b) Thermogravimetric results obtained for PDLLA (dotted line) and the PDLLA/ $\alpha$ CD IC (solid line); the dashed lines were used to calculate the onset temperatures.

stability of such an organization was assessed from WAXS measurements during heating experiments using synchrotron radiation (see WAXS patterns in Figure 1a). During heating, the peaks shift to lower angles because of the dilatation of the unit cell. Above $\approx 320^{\circ} \mathrm{C}$ one can detect a systematic deterioration of the diffraction peaks, which indicates the loss of crystallinity. A similar behaviour is found in semi-crystalline polymers, which loose their crystalline structure across the melting temperature. However, for the IC no phase transition is associated with the disruption of the initial organization, but in this case is a simple consequence of thermal degradation. In Figure $1 b$, TGA curves are presented for both PDLLA and PDLLA $/ \alpha C D$. IA strong mass loss with an onset at 302 and $303^{\circ} \mathrm{C}$ is clearly seen for the two materials, respectively. We can conclude that the inclusion process with $\alpha$-CD does not influence significantly the thermal stability of PDLLA.
Moreover, the range of temperatures where thermal degradation of PDLLA $/ \alpha C D$ takes place is in the same region at which the diffraction peaks disappear. Therefore, it can be concluded that the obtained IC is structurally stable up to quite high temperatures, degradation of the supramolecular organization is essentially dictated by the thermal stability of the material. This behaviour could not be observed in regular semi-crystalline polymers and may have practical relevance. For example, it was shown that inclusion complexes of $\alpha$-CD and aliphatic polymers could be used a nucleating agents, and may greatly accelerate the nucleation and crystallization of semi-crystalline polymers. ${ }^{[20]}$ We may also envisage new uses of PDLLA/ $\alpha \mathrm{CD}$-based materials in biomedical applications, where the degradation profile of PDLLA could be greatly affected and where bioactive molecules or therapeutic molecules could be associated.

The glass transition of the materials was investigated by DSC. Figure 2 shows the scans obtained after ageing at $40^{\circ} \mathrm{C}$ for $14 \mathrm{~d}$. For the case of PDLLA the presence of an endothermic peak at $57^{\circ} \mathrm{C}$, which is associated to enthalpy recovery of the material that was previous subjected to an ageing process where structural relaxation took place, is clearly seen. The second heating scan reveals the presence of the glass transition in the same temperature range, which is now detected by a typical step change in the heat flow curve, assigned to the jump in the heat capacity, $C_{\mathrm{p}}$. For PDLLA $/ \alpha$ CD IC no glass transition seems to occur even after 2 weeks of ageing. This could be explained by the limited mobility of the polymeric chains in the channel structure of the IC (see scheme in Figure 2). A jump in $C_{p}$ analogous to a glass transition implies a change in the temperature dependence of the number of microstates $\Omega$ available for the polymeric chains, as deduced from the Boltzmann's formula $S=k \cdot \ln \Omega$, and $C_{\mathrm{p}}=T \cdot \mathrm{d} S / \mathrm{d} T=$ $k T \cdot d \ln \Omega / d T$, where $S$ is the entropy and $k$ is the Boltzmann constant. This occurs during the glass transition in amorphous systems because of the exponential increase

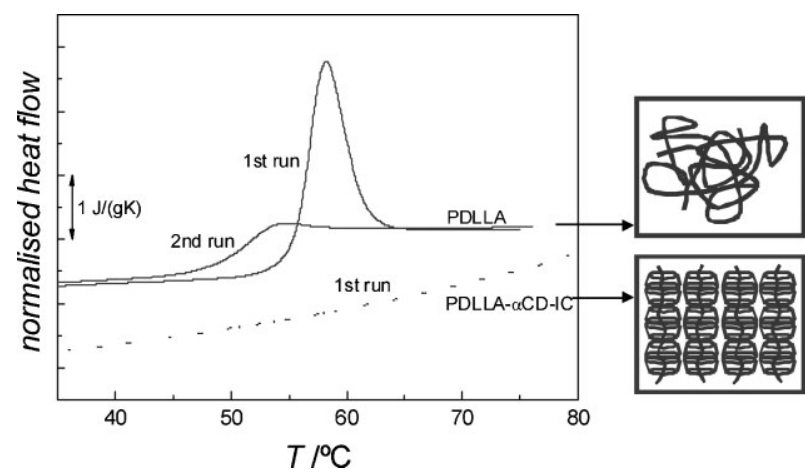

Figure 2. DSC scans of PDLLA and PDLLA $/ \alpha C D$ after an ageing period at $40^{\circ} \mathrm{C}$ for 2 weeks. The second run for the case of PDLLA is also shown. 
of the number of configurational states with increasing temperature in the liquid state. Any segmental mobility that takes place in the IC does not involve such significant variations in the number of microstates, and thus the heat capacity is not expected to experience a considerable variation. A similar behaviour was observed for a relaxation process found in a liquid crystalline polymer that involved mobility of stiff groups: no signal was detected by DSC but a relaxation process could be found by DMA. ${ }^{[21]}$

DMA tests were performed on the materials (see Figure 3). In this case, the $\alpha$-relaxation could be identified in both cases. The loss factor peaks of the IC seem to be broader than in PDLLA. In both cases the loss peaks shift to higher temperatures with increasing frequency. This dependency was used to build a relaxation plot, where the temperature corresponds to $T_{\max , \tan \delta}$ for each frequency, $f$. The behaviour at $1 \mathrm{~Hz}$ for both materials indicates a temperature difference of about $15^{\circ} \mathrm{C}$ for the occurrence of the glass transition, which evidences the

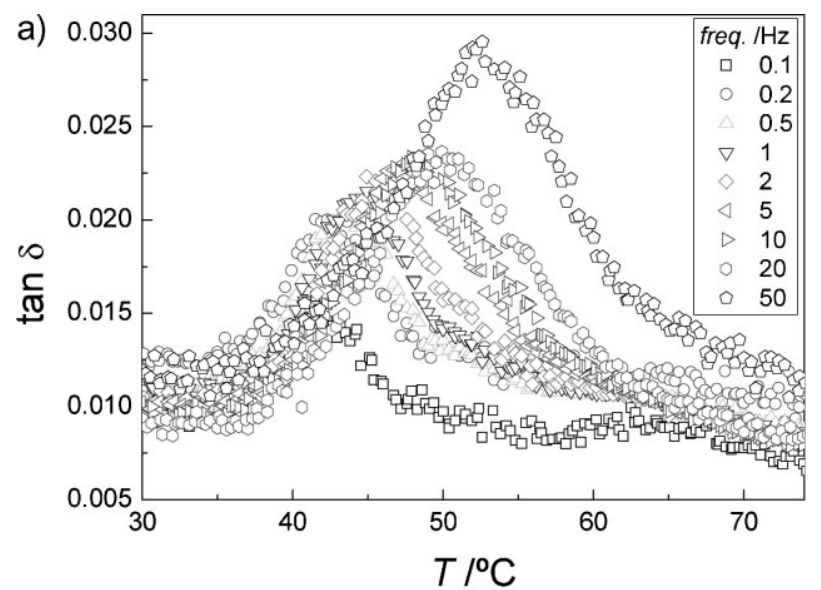

b)

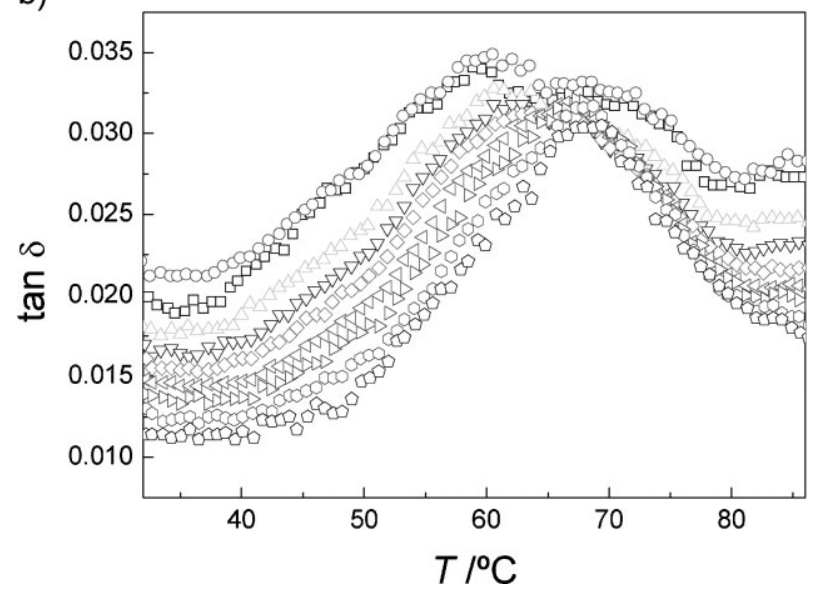

Figure 3. Loss factor at different frequencies (see legend) for a PDLLA film (a) and a PDLLA $\alpha$ CD IC powder (b) placed in metallic pockets. strong confinement as a result of the IC organization. For comparison, note that the confinement effect produced by the crystalline lamellae in semi-crystalline PLLA leads to an increase in $T_{\mathrm{g}}$ of about $6^{\circ} \mathrm{C}^{[15]}$ For the semi-crystalline case the mobile amorphous phase is confined between the crystalline lamellae and exhibits thicknesses of the order of $6-7 \mathrm{~nm} ;{ }^{[22]}$ for the case of the ICs, the PDLLA chains adopt a much more aligned configuration, where they must pass through the cavities of the $\alpha$-CDs, with just $0.49 \mathrm{~nm}$ diameter, which are also organized in a supramolecular structure. Therefore, the confinement effect found in the IC is highly superior to the one in semi-crystalline polymers. For the case of PDLLA a clear curvature is found. The results were fitted according to the Vogel-Fulcher-Tamman-Hesse equation (VFTH): ${ }^{[23]}$

$$
\tau(T)=\tau_{0} \exp \frac{B}{T-T_{0}}, \quad T_{0}<T_{\mathrm{g}}
$$

where $\tau_{0}$ is a pre-exponential factor and $B$ and $T_{0}$ are specific adjustable material parameters. The values of $\tau(T)$ were obtained from the frequency of the experiments: $\tau=1 /(2 \pi f)$. The best fit yielded the following adjustable parameters: $\ln \tau_{0}(\mathrm{~s})=-22.5 \pm 5.7 ; B=681 \pm 385 \mathrm{~K}$, and $T_{0}=285.0 \pm 9 \mathrm{~K}\left(R^{2}=0.995\right)$ (see solid line in Figure 4). The VFTH behaviour was not found in the relaxation plot of PDLLA $/ \alpha C D$ IC, which exhibited a more linear relationship. Such trend suggests that the IC behaves as a 'strong' system, according to the fragile/strong classification proposed by Angell, ${ }^{[24]}$ whereas the amorphous PDLLA exhibits a more fragile nature. Assuming an Arrhenius dependency for PDLLA $/ \alpha C D$, where $\ln \tau \propto E_{\mathrm{a}} / R T$, where $E_{\mathrm{a}}$ is the activation energy and $R$ is the ideal gas constant, we obtained from the linear regression $E_{\mathrm{a}}=580 \pm 44 \mathrm{~kJ} \cdot \mathrm{mol}^{-1}$

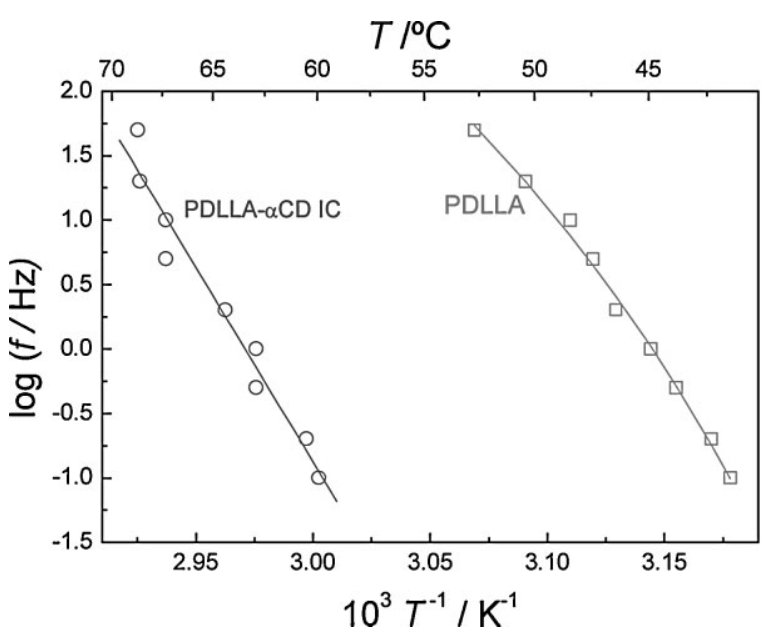

Figure 4. Relaxation map for PDLLA (squares) and PDLLA/ $\alpha$ CD IC (circles), obtained from the data shown in Figure 3, with the corresponding VFTH and Arrhenius fits, respectively (solid lines). 
$\left(R^{2}=0.96\right)$. Such a value is higher than the activation energies observed for the glass transition of semicrystalline PLLA obtained using mechanical spectroscopies, which were typically lower than $400 \mathrm{~kJ} \cdot \mathrm{mol}^{-1}$. ${ }^{25]}$ Therefore, we can conclude that the confinement effect exerted by the organized arrangement at the sub-nanometre level in the IC leads to different glass transition dynamics than that found in the bulk state or even in semi-crystalline systems. More experiments should be done in ICs with other amorphous polymers in order to elucidate the nature of the conformational mobility in such supramolecular systems. For example, complementary information could be obtained if a broad frequency range could be used in the characterisation of the $\alpha$-relaxation of these materials, using, for example, dielectric relaxation spectroscopy.

\section{Conclusion}

In summary an IC was produced that combined $\alpha-C D$ and amorphous PDLLA which exhibited a channel-like crystalline organization. Such a supramolecular structure was maintained up to the thermal degradation of the material, opening new perspectives for the applicability of such nanostructured systems. The glass transition dynamics in the IC were investigated by DMA. This enabled the corresponding relaxation plot to be obtained, which exhibited an Arrhenius trend, quite different to the one observed in PDLLA, characterized by a typical VFTH behaviour and located at lower temperatures.

Acknowledgements: This work was financial supported by $F C T$, through the POCTI and FEDER programmes, and POCTI/FIS/61621/ 2004. The experimental help of Mr. R. Picciochi and Miss A. Pinheiro is also acknowledged.

Received: March 24, 2008; Revised: May 13, 2008; Accepted: May 14, 2008; DOI: 10.1002/marc. 200800180

Keywords: biodegradable; crystal structure; glass transition; supramolecular structures; thermal properties
[1] [1a] J. M. Lehn, Science 2002, 295, 2400; [1b] J. M. Lehn, Proc. Natl. Acad. Sci. U. S. A. 2002, 99, 4763.

[2] G. Wenz, Angew. Chem. Int. Ed. Engl. 1994, 33, 803.

[3] A. Harada, M. Kamachi, Macromolecules 1990, 23, 2821.

[4] T. Mori, T. Dong, K. Yazawa, Y. Inoue, Macromol Rapid Commun. 2007, 28, 2095.

[5] T. Dong, W. Kai, P. Pan, A. Cao, Y. Inoue, Macromolecules 2007, 40, 7244 .

[6] X. Shuai, M. Wei, F. E. Porbeni, T. A. Bullions, A. E. Tonelli, Biomacromolecules 2002, 3, 201.

[7] Y. Ohya, S. Takamido, K. Nagahama, T. Ouchi, T. Ooya, R. Katoono, N. Yui, Macromolecules 2007, 40, 6441.

[8] D. M. Xie, K. S. Yang, W. X. Sun, Curr. Appl. Phys. 2007, 7S1, e 15.

[9] C. M. Patist, M. B. Mulder, S. E. Gautier, V. Maquet, R. Jerome, M. Oudega, Biomaterials 2004, 25, 1569.

[10] K. S. Soppimath, T. M. Aminabhavi, A. R. Kulkarni, W. E. Rudzinski, J. Controlled Release 2001, 70, 1.

[11] M. Wei, A. E. Tonelli, Macromolecules 2001, 34, 4061.

[12] Y. Paik, B. Poliks, C. C. Rusa, A. E. Tonelli, J. Schaffer, J. Polym. Sci., Part B: Polym. Phys. 2007, 45, 1271.

[13] T. A. Bullions, M. Wei, F. E. Porbeni, M. J. Gerber, J. Peet, C. M. Balik, A. E. Tonelly, J. Polym. Sci., Part B: Polym. Phys. 2002, 40, 992.

[14] M. Alcoutlabi, G. B. McKenna, J. Phys. Condens. Matter 2005 17, R461.

[15] J. F. Mano, J. L. Gómez Ribelles, N. M. Alves, M. Salmerón Sanchez, Polymer 2005, 46, 8258.

[16] Y. Wang, J. L. Gómez-Ribelles, M. Salmerón Sánchez, J. F. Mano, Macromolecules 2005, 38, 4712.

[17] [17a] M. Dionísio, M. T. Viciosa, Y. Wang, J. F. Mano, Macromol. Rapid Commun. 2005, 26, 1423; [17b] R. Brás, M. T. Viciosa, Y. Wang, M. Dionísio, J. F. Mano, Macromolecules 2006, 39, 6513.

[18] A. Harada, M. Kamachi, Macromolecules 1990, 23, 2821.

[19] J. W. Chung, T. J. Kang, S.-Y. Kwak, Macromolecules 2007, 40, 4225.

[20] T. Dong, Y. He, B. Zhu, K.-M. Shin, Y. Inoue, Macromolecules 2005, 38, 7736.

[21] J. F. Mano, J. L. Gómez Ribelles, Macromolecules 2003, 36 2816.

[22] R. Picciochi, Y. Wang, N. M. Alves, J. F. Mano, Colloid Polym. Sci. 2007, 285, 575.

[23] [23a] H. Vogel, Phys. Z. 1921, 22, 645; [23b] G. A. Fulcher, J. Am. Chem. Soc. 1925, 8, 339; [23c] G. Tamman, W. Z. Hesse, Anorg. Allg. Chem. 1926, 156, 245.

[24] [24a] C. A. Angell, Science 1995, 267, 1924; [24b] P. G. Debenedetti, F. H. Stillinger, Nature 2001, 410, 259.

[25] J. F. Mano, Macromol. Biosci. 2005, 5, 337. 